\section{Moving towards a graphene world}

Welcome to graphene: the flat carbon sheet with revolutionary aspirations. This thinnest possible pencil-lead shaving has already interested theoretical physicists with its electronic properties, and is predicted to edge aside silicon in the microchips of the future. Now it's ready for its first practical application.

Carbon exists in many forms: 'buckyballs', diamond, nanotubes and graphite to name a few. In 2004, André Geim of the University of Manchester, UK, together with Russian colleagues, created another form - carbon layers just one atom thick, called graphene (K. S. Novoselov et al. Science 306, 666-669; 2004). Physicists have since documented some remarkable properties of this material. But industrial applications have seemed some way off, as graphene

"Nothing is an
insurmountable
problem. All the
lights are still go."

tronic properties make graphene a candidate to replace silicon in a fresh era of microchip electronics. "Graphene is quite different from conventional semiconductors such as silicon," explains Philip Kim, a physicist from Columbia University in New York. Electrons move though silicon in a series of collisions; these generate heat and limit the speed and size of silicon transistors. The density of components on silicon chips has doubled every 18 months or so since the 1960 s, a trend known as 'Moore's law' after Intel's co-founder Gordon Moore predicted it in 1965. But many believe silicon chips will soon reach their limit.

In graphene, however, electrons shoot along with minimal resistance. This, says Kim, may allow for low-power, faster-switching has proved difficult to produce on a large scale. It was made by unwieldy methods such as rubbing flecks off a piece of graphite, as Geim did, or boiling up silicon carbide in a vacuum.

Now, Rodney Ruoff and his team at Northwestern University, Illinois, have come up with a way to make large amounts of graphene embedded in a polymer matrix. The researchers start with graphite oxide graphite with oxygen-containing chemical groups attached. After further chemical modification and treatment with ultrasound, the material separates into layers, and disperses through a solvent in which a polymer such as polystyrene is also dissolved. Chemical reduction removes most of the oxygen groups, and removing the solvent leaves behind graphene sheets crumpled within the solid polymer (see pages 282 and 254).

The resulting material is strong, and electrically and thermally conducting. Such properties are similar to those of carbon nanotube composites, which isn't surprising as a sheet of graphene is basically an unrolled nanotube. But the graphene-based composites produced by Ruoff's chemical method are cheaper and more reliable to manufacture, so could be perfect for things that need to be lightweight, strong and conducting, such as aircraft fuselages.

Although graphene's first foray into industry is likely to be as a composite, several groups are working on unlocking the potential of the isolated sheets. For one thing, the sheets' elec- transistors. "But it's difficult to turn graphene off, which will make it hard to use in an electrical switch," he notes. "Really, we need new types of electronic architecture to make best use of graphene."

\section{Trumping silicon}

Also enthusiastic about graphene's potential in computing is Walt de Heer, of Georgia Tech's School of Physics in Atlanta. His team, with French collaborators at the National Centre of Scientific Research in Grenoble, is attempting to etch electronic structures into layers of graphene wafers grown on silicon carbide. He points out that carbon nanotubes would give the same electronic advantages as graphene sheets but that it is difficult to produce them in large amounts and organize them on a chip, and that high electrical resistances occur when nanotubes are connected with metal wires in an electronic circuit.

"No one sees nanotubes as a serious avenue," says de Heer. In contrast, flat graphene wafers are easily etched using conventional lithography techniques. De Heer also hopes that connecting wires won't be necessary. He envisages future electronic circuits made up of continuous graphene sheets, saying that cutting graphene into ribbons of different widths controls its conducting properties.

De Heer, who along with his team has

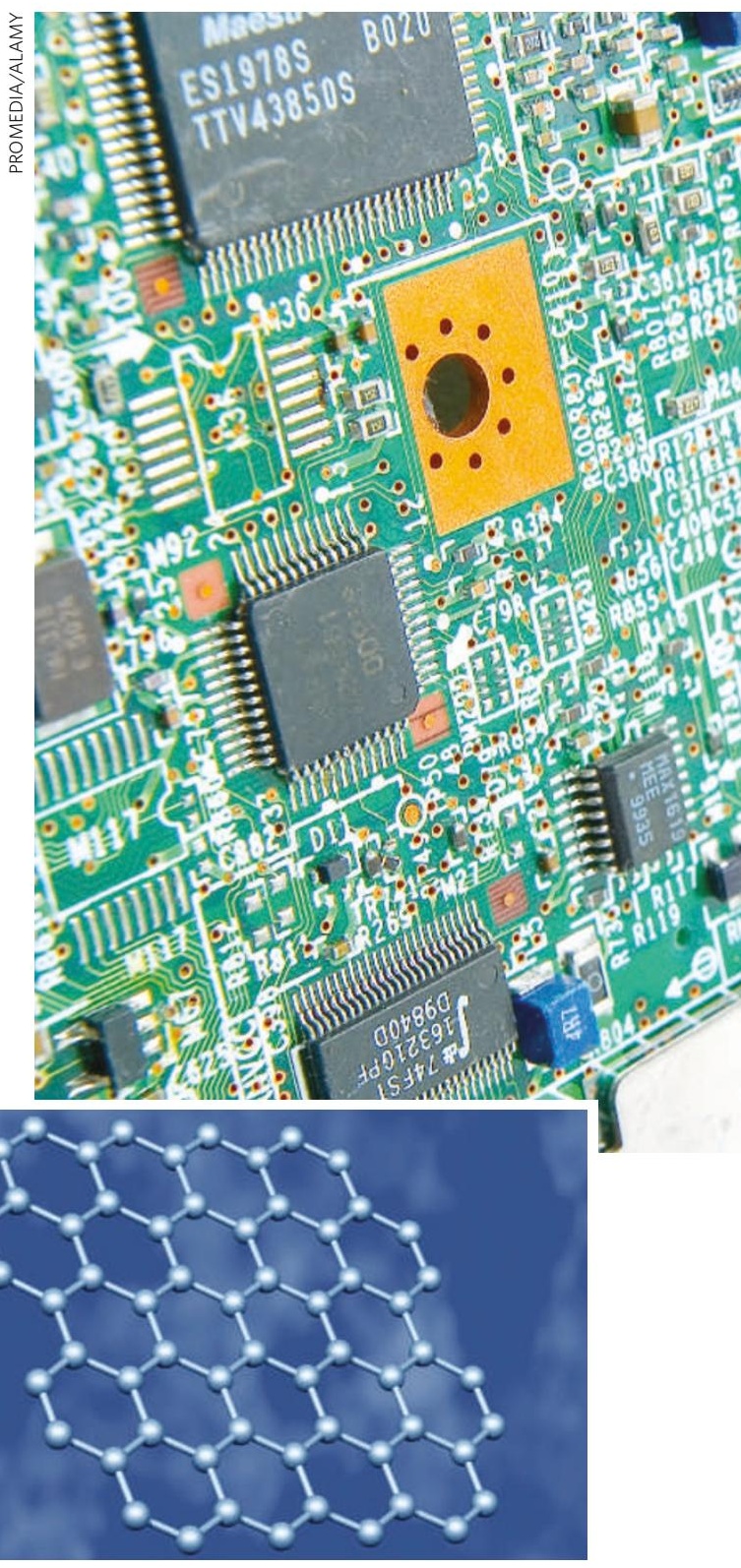

attracted funding from Intel, is confident in graphene's electronic potential, although he cheerfully predicts that the revolution won't come just yet. "Industry has no reason to switch over from silicon until the end of Moore's law in 10 or 15 years," he says. Geim is more wary. "Electronic applications require reliable largescale production of graphene," he says. "At the moment the quality is rather mediocre."

Geim is more excited about graphene's usefulness as a research tool. The carbon lattice in the sheets is extremely regular, giving the electrons within the lattice unusual properties. "The electrons move collectively in a manner that mimics particle behaviour at close to light speed," explains Geim. He and other teams are using graphene to investigate quantum 
